\title{
LA PERCEPCIÓN DE LOS NIVELES DE AUTONOMÍA PROFESIONAL DE LOS PERIODISTAS DE ECUADOR
}

\section{The Perception of Professional Autonomy of Ecuador's Journalists}

\section{A percepção dos níveis de autonomia profissional dos jornalistas do Equador}

\author{
OLLER, Martín. Universidad de las Américas (UDLA) (Ecuador) \\ martin.olleralonso@gmail.com
}

CHAVERO, Palmira. Facultad Latinoamericana de Ciencias Sociales (flacSO) (Ecuador) pchavero@gmail.com

ORTEGA, Edith. Universidad Central del Ecuador (Ecuador) edithkaterine@yahoo.com

Fecha de recibido: 02 de septiembre de 2015

Fecha de aceptado: 27 de octubre de 2015

\section{RESUMEN}

Este artículo muestra los resultados obtenidos sobre el nivel de autonomía profesional que los periodistas ecuatorianos perciben en su trabajo diario y los principales limitantes que encuentran basados en los niveles o factores de influencia. Este estudio se enmarca dentro del proyecto Cultura Periodística de Ecuador (CPE) en el que se realizaron 31 entrevistas en profundidad a periodistas en activo de 6 medios de comunicación nacionales (públicos, privados y comunitarios). La autonomía profesional es uno de los principales valores que determinan el nivel de profesionalización del periodismo y el profesionalismo de los periodistas al estar directamente vinculada a los conceptos de autorregulación, libertad de expresión e independencia. Esta situación provoca que las influencias percibidas por los 
periodistas - procedentes de los niveles de actor, institución y sistemas (Oller \& Meier, 2012) - afecten directamente a su libertad de acción profesional y, por lo tanto, a sus índices de autonomía. Los resultados muestran que la mayor parte de los periodistas entrevistados considera que posee total libertad en su medio de comunicación y que los principales limitantes proceden del nivel del sistema, concretamente de la actual legislación en comunicación.

Palabras clave: Cultura periodística, autonomía profesional, factores de influencia, América Latina, Ecuador.

\begin{abstract}
This article shows the results about the perception of the level of professional autonomy of Ecuadorian journalists in their daily work and major constraints that are based on influence factors. This study is part of the project Culture Journalism of Ecuador (CJE) in which 31 journalists within 6 media (public, private and communitarian) were interviewed. Professional autonomy is one of the main values that determine the level of professionalism in journalism, which is directly linked to the concepts of self-regulation, freedom of speech and independence. This situation causes that influences perceived by the journalists - from the actor level, institution level and system level (Oller \& Meier, 2012) - directly affect their professional freedom of action and, therefore, their rates of autonomy. The results show that most of the journalists interviewed considered to have complete freedom in their media and that the main constraints come from the system levels, specifically the current legislation in communication.
\end{abstract}

Keywords: Journalistic culture, Professional autonomy, Influence factors, Latin America, Ecuador.

\title{
RESUMO
}

Este artigo mostra os resultados obtidos sobre o nível de autonomia profissional que os jornalistas equatorianos percebem em seu trabalho diário e os principais limitantes que encontram baseados nos níveis ou fatores de influência. Este estudo enquadra-se dentro do projeto Cultura Jornalística do Equador (CPE - Cultura Periodística de Ecuador) no qual se realizaram 31 entrevistas em profundidade a jornalistas de 6 meios de comunicação nacionais (públicos, privados e comunitários). A autonomia profissional é um dos principais valores que determinam o nível de profissionalização do jornalismo e o profissionalismo dos jornalistas ao estar diretamente vinculada aos conceitos de auto regulação, liberdade de expressão e independência. Esta situação provoca que as influências percebidas pelos jornalistas, procedentes dos níveis de ator, instituição e sistemas (Oller \& Meier, 2012), afetem diretamente a sua liberdade de ação profissional e, portanto, a seus índices de autonomia. Os resultados mostram que a maior parte dos jornalistas entrevistados considera que possui total liberdade em seu meio de comunicação e que os principais limitantes procedem do nível do sistema, concretamente da atual legislação em comunicação.

Palavras-chave: Cultura jornalística, autonomia profissional, fatores de influência, América Latina, Equador. 


\section{Introducción}

Este artículo muestra un mapa aproximativo de la percepción de la autonomía profesional que los periodistas tienen en Ecuador, analizando los límites que estos encuentran dentro de la cultura periodística del país. La autonomía profesional es uno de los principales valores que determina el nivel de profesionalización del periodismo y el profesionalismo de los periodistas al estar directamente vinculada con los conceptos de autorregulación, libertad de expresión e independencia. Este estudio, siguiendo la línea planteada por Mellado y Humanes (2012), intenta entender el concepto de autonomía profesional a partir de una idea multidimensional donde los periodistas perciben su nivel de autonomía como un factor indispensable dentro de la práctica profesional en el que influyen factores individuales, organizacionales y contextuales.

El estudio de la autonomía periodística ha sufrido un giro; tradicionalmente analizada con base en los valores y prioridades impuestas a los periodistas dentro de su entorno organizacional (p.e. Elliott, 1972; Schlesinger, 1978), actualmente se vincula a la separación de los medios y el Estado (Sjøvaag, 2013, p. 163) y "al riesgo de una posible instrumentalización política" (Aznar, 2011, p. 150) o al efecto contrario de mediatización (Mazzoleni \& Schulz, 1999; Chavero et al., 2013). Esta situación ha propiciado que la autonomía periodística se plantee como un punto clave del desarrollo del periodismo como profesión (Johnstone et al., 1976).

El proceso de cambio en el que se encuentra Ecuador desde la llegada a la presidencia de Rafael Correa en 2007 se caracteriza por la rápida transformación que se está produciendo en la regulación y la estructuración del sistema mediático del país. Tal es así que, como afirma Hervieu y Samson (2010) en el informe de Reporteros sin Fronteras, en Ecuador existe un "ambiente mediático volcánico" (p. 2).

A partir de los resultados obtenidos en el proyecto Cultura Periodística de Ecuador (CPE) se han establecido los parámetros que definen la cultura periodística ecuatoriana con base en 1) el estudio de las ideas, las percepciones y las opiniones que los entrevistados tienen sobre sus límites profesionales, su grado de libertad de expresión, los niveles de censura y la percepción de autonomía profesional - se llevaron a cabo 31 entrevistas en profundidad-; 2) el análisis contextual del sistema político, social, económico y organizacional de los medios de comunicación y el sistema mediático, y 3) la evaluación de las influencias percibidas por los periodistas procedentes de los niveles de actor, institución y sistemas (Oller \& Meier, 2012).

Los objetivos de esta investigación son: 1) Establecer el nivel de autonomía profesional que los periodistas entrevistados perciben dentro de la cultura periodística de Ecuador; 2) Conocer el grado de decisión que poseen los periodistas entrevistados en el proceso de preparación, elaboración y publicación de sus trabajos en las redacciones de los medios de comunicación de Ecuador, y 3) Determinar las influencias percibidas por los periodistas entrevistados procedentes de los factores contextuales que determinan sus niveles de autonomía dentro de la cultura periodística de Ecuador.

\section{Cultura periodística de Ecuador}

"La cultura periodística construye los rasgos identitarios de los roles y de las rutinas que, en un plano abstracto, representan el marco simbólico de un colectivo" (Oller \& Barredo, 2013, p. 10). Por lo tanto, la actividad profesional 
de los periodistas queda determinada por la cultura periodística en la que se encuentran. Como afirma Hanitzsch (2007), esta "es la idea específica sobre la que el periodista evalúa y ordena sus ideas culturales, con la que crea su propia realidad significativa dentro de un grupo" (p. 373).

Hoy en día, el denominado periodismo globalizado está traspasando las fronteras nacionales y culturales (Löffelhoz \& Weaver, 2008, p. 3) ya que el carácter global de la cultura periodística occidental, que ha actuado hasta ahora como epicentro, comienza a remitir debido a la aparición de las culturas periodísticas híbridas que comparten características identitarias propias y otras adoptadas de modelos estandarizados.

La cultura periodística de Ecuador queda encuadrada dentro de las culturas periodísticas intermedias (Oller \& Barredo, 2013) que difieren del estándar occidental, establecen que el periodismo se muestra como un campo social interpretativo de la comunidad en la que se encuentra, con sus propias reglas y discursos, apareciendo en una situación de constante negociación y relativización con respecto de las diferentes perspectivas culturales (Zelizer, 2009).

En Ecuador, la primera década del nuevo milenio supuso un cambio sustantivo de todos los niveles -social, político, económico y mediático-. Como Murillo-Ruiz (2011) confirma,

a partir de 2005 los partidos políticos, de izquierda y de derecha, sufren un colapso del que aún no se recupe-

ran. La emergencia de un discurso refundador de la patria fue cobrando fuerza y quienes lo abanderaron fueron

precisamente aquellos movimientos ciudadanos que le apostaban a otro modo de ejercer la política" (p. 177).

Esta coyuntura llevó a un cambio radical y a que, en 2007, Rafael Correa asumiera la presidencia de la República y comenzara con su denominada "Revolución Ciudadana.

En política comunicacional, la profunda crisis económica en Sur América y la crisis bancaria en Ecuador de 1999-2000 propiciaron que los vínculos entre el poder financiero del país y los medios de comunicación se acentuaran (Checa-Godoy, 2012; Flor, 2013). Por este motivo, el Gobierno de Rafael Correa apostó desde un principio por una reestructuración del sistema mediático debido a que hasta entonces en el país un pequeño grupo de propietarios empresariales del poder simbólico (y mediático), que actuó como legitimador de los valores profundos del neoliberalismo y se posicionó como único defensor de la libertad de expresión, estaban ubicados intersticialmente entre los poderes políticos y económicos (Reyes, 2014). Para llevar a cabo este proceso, en la Constitución aprobada en 2008 se tomaron medidas para separar el poder mediático del financiero y se creó un compromiso para una legislación en el área de comunicación que se hizo realidad - tras un proceso de debate asambleario de cuatro años- el 25 de junio de 2013 en el que fue aprobada la Ley Orgánica de Comunicación del Ecuador (Loc). Esta fue la propuesta gubernamental de mayor relevancia en busca de una democratización de la comunicación, siguiendo la línea propuesta en otros países latinoamericanos como Argentina, Venezuela, Bolivia y Uruguay ${ }^{1}$.

A estos aspectos se unieron: 1) el fortalecimiento de las instituciones públicas en materia comunicativa²; 2) el reordenamiento del mercado mediático en busca del desarrollo de los medios de servicio público y comunitarios

1 En 2014 países como México y Brasil aprobaron nuevas leyes en materia de comunicación y en Brasil, Paraguay y Perú están en pleno debate.

2 El Consejo Nacional de Telecomunicaciones (CONATEL), la Superintendencia de Telecomunicaciones (superTEL), el Ministerio de Telecomunicaciones y Sociedad de la Información (MINTEL), la Secretaría Nacional de Comunicación (sEcom). Tras la aprobación de la Ley Orgánica de Comunicación se crearon la Superinten- 
apoyado en los principios de universalidad, diversidad, independencia y especificidad (Análisis del Desarrollo Mediático en Ecuador, 2011), produciéndose un fortalecimiento de los medios comunitarios -en 2010 solo existían en Ecuador dos emisoras de radio reconocidas como comunitarias y ningún canal de televisión (Checa-Godoy, 2011)-y creándose la Radio Pública del Ecuador (2008), el canal de televisión Ecuador Tv (2008), el diario El Telégrafo (2008), el diario El Ciudadano (2008), el diario PP El Verdadero (2010) y la Agencia Nacional de Noticias de Ecuador y Sudamérica (Andes) (2009)33 3) la redistribución equitativa del espectro radioeléctrico de frecuencias en el que el 33\% queda para los medios públicos, el $33 \%$ para los medios privados y el $34 \%$ para los medios comunitarios ${ }^{4}$ y 4) el incremento del gasto público en publicidad oficial e institucional en los medios de comunicación ${ }^{5}$.

A pesar de la firme apuesta del Estado por cambiar el paisaje mediático ecuatoriano, aún hoy en día el $91 \%$ de los medios de comunicación son de propiedad privada, el $5 \%$ públicos y el $4 \%$ comunitarios $^{6}$, además, el $72 \%$ de los periodistas trabaja en medios privados (соRDicom, 2014). De forma que, aunque el oficialismo desarrolla una política basada en la democratización de la información, la reconfiguración del campo mediático, el desarrollo de nuevas prácticas informativas (Abad, 2013) y que "durante estos años de gestión el presidente ha cuestionado insistentemente las condiciones de producción de los medios y ha evidenciado sus vínculos comerciales, financieros y políticos con otros sectores tradicionales de poder" (Ramos, 2013, p. 71), actualmente existe una polarización del discurso que plantea un enfrentamiento Gobierno/medios de comunicación masivos, en el que ambos intentan apropiarse de la producción del espacio público (Reyes, 2014) y del control del relato social, situación que ha creado un marco de incertidumbre (Abad, 2013).

\section{La autonomía como valor profesional del periodismo}

La autonomía es el derecho de autogobernación, donde "el estado de autogobierno se caracteriza por la capacidad de independencia, soberanía, jurisdiccionalidad” (Sjøvaag, 2013, p. 155) y “autorregulación” (Sjøvaag, 2013, p. 163). En la misma línea, autores como Merrill (1974) y Beam (1990) definen la autonomía profesional como la capacidad de criterio o la posibilidad de tomar decisiones personales sin ser afectados por las influencias externas o internas; lo anterior esta asociado con el pluralismo y la defensa de la función social de la profesión en contra de las restricciones de la libertad de expresión y los intereses públicos (McQuail 1994, citado por Mellado \& Humanes, (2012)). De modo que, para algunos autores, en el periodismo como profesión "son imprescindibles mecanismos que protejan y garanticen en la redacción esa independencia y autonomía del periodista” (Aznar, 2011, p. 174), ya que la autonomía implica una autogestión de las técnicas profesionales (McDevitt, 2003). Esta capacidad de los

dencia de la Información y Comunicación (SUPERCom) y el Consejo de Regulación y Desarrollo de la Información y Comunicación (CORDICOM).

3 A la puesta en marcha de estos nuevos medios de comunicación se une la creación de espacios mediáticos destinados a transmitir la información gubernamental. El más conocido es: Enlace Ciudadano (también conocido como "la sabatina"), emitido todos los sábados en directo a través de todos los medios públicos.

4 Art. 106 de la Ley Orgánica de Comunicación.

5 La publicidad oficial en 2008 supuso a las arcas del gobierno 17 millones de dólares, en 2009 llegó a los 40 y en 2010 superó los 60 millones (Checa-Godoy, 2011).

6 Según el Registro Único de Medios del cordicom. 
periodistas de frenar los intentos de presión empresarial es, para algunos autores, el principal poder de la profesión periodística frente a los medios de comunicación (Ortega \& Humanes, 2001).

Por lo tanto, se entiende que en una democracia liberal los medios de comunicación son "libres de publicar lo que quieran y critiquen libremente, e incluso ataquen, al gobierno con el propósito de mejorar la sociedad" (Rettberg \& Rincón, 2012, p. 21). Bajo esta perspectiva, "para que esto ocurra de manera democrática, los periodistas y las entidades periodísticas deben ser autónomos en sus decisiones respecto a la cobertura de las noticias y críticas al gobierno" (Flor, 2013, p. 3). De modo que los conceptos cohesivos de la idea de 'estado de democracia liberal' que la política en cualquier país debe intentar establecer son: 1) un alto grado de libertad de las personas, que incluye la garantía de derechos como la libertad de expresión y el derecho de asociación y 2) un sistema de gobierno definido por su competencia, la participación ciudadana y su capacidad de respuesta (O’Neil, 2010). Bajo estas premisas, la profesión periodística de un país queda definida por el nivel de libertad de prensa, "entendido como el resultado del paralelismo político de los medios y la intervención del Estado” (Reich y Hanitzsch, 2013, p. 140).

A pesar de que el modelo de responsabilidad social se establece con el fin de garantizar una cobertura informativa más equitativa, los factores económicos privados siguen siendo la fuerza dominante cuando se trata de llevar a cabo la cobertura de las noticias (Punín, 2011). Si se tiene en cuenta que los medios de comunicación privados tienen la necesidad de sobrevivir a través de los ingresos provenientes de la publicidad, los países donde la legislación vigente en comunicación se sustenta en un modelo mediático basado en la responsabilidad social (Ecuador, Venezuela, Bolivia, Argentina, Uruguay, etc.) se convierten en objeto de dos tipos de censura o control: 1) la censura impuesta por el Estado, que busca garantizar el equilibrio en la cobertura y 2) la censura que proviene de los intereses privados de los propietarios de los medios de comunicación (Rettberg \& Rincón, 2012). Estas amenazas a la libertad de expresión y de prensa son situaciones que deben preocupar a todos: Gobierno, sociedad civil, periodistas y ciudadanos de a pie, entre otros, porque van directo al corazón de la democracia (Ricaurte, 2010).

Según Reese (1999), a finales del siglo pasado, el trabajo de los periodistas en las redacciones se basaba en la adhesión a un código de conducta, a la organización del medio y a los grupos profesionales que apoyaban la actividad profesional. En este punto se diferenciaba de actividades como el derecho, la medicina o la ingeniería, profesiones con una base común y una experiencia compartida en la universidad. Este tema planteado por el autor es uno de los más importantes y debatidos desde la segunda mitad del siglo xx debido a su relación con el proceso de profesionalización de la actividad periodística que tiene presente en todo momento "la formación académica y el tipo de educación o formación profesional recibida” (Johnstone et at., 1976, p. 123).

Hallin y Mancini, en su trabajo Comparing Media Systems: Three Models of Media and Politics de 2004, determinaron que el modelo mediático de un país está formado por un conjunto de factores entre los que se encuentran el grado de autonomía y el profesionalismo de los periodistas - poder de las asociaciones de profesionales, regulación de acceso a la profesión (grado de intrusismo) y nivel de formación-.

Los periodistas tradicionalmente defienden la autonomía como el principio que permite que los medios cumplan con su deber de informar a la ciudadanía de forma libre de sesgo partidista y otras influencias corruptoras. Desde este punto de vista, los periodistas sirven al público mediante la búsqueda, no en un sentido estrictamente epistemológico, del equilibrio, la equidad y la objetividad (McDevitt, 2003, p. 156). 
Perspectiva que asociaría la autonomía profesional a la idea de la función de servicio público de los medios de comunicación, muy relacionada con la propuesta del Gobierno de Rafael Correa, apoyado en la Loc del 25 de junio de 2013 (Chavero \& Oller, 2014).

En Ecuador esta profesionalización del periodismo se sustenta en factores como la educación o la formación, y en la autonomía del profesional. Respecto a la formación del profesional destacan la aprobación y la proliferación de nuevas facultades y escuelas de Comunicación Social y Periodismo y carreras técnicas y tecnológicas (Oller \& Chavero, 2015a) y la certificación de los perfiles ocupacionales de las personas que ejercen esta actividad (CORDICOM, 2014) $)^{7}$, reconociendo formalmente las competencias de los trabajadores a través de la expedición de una certificación que confirma documentalmente la competencia del trabajador por parte del Organismo de Evaluación de la Conformidad ${ }^{8}$, la SENESCYT ${ }^{9}$, el SECAP ${ }^{10}$ y la SETEC $^{11}$ (Oller \& Chavero, 2015a). Relacionado con la autonomía en la profesión, un profesional es quien pone especial énfasis en su servicio, en su actividad intelectual, en su autonomía, en su influencia y está en posesión de técnicas intelectuales especializadas que permiten un servicio eficaz a la comunidad (Carr-Saunders \& Wilson, 1933; Aldridge \& Evetts, 2003; Oller \& Meier, 2012; Oller \& Chavero, 2015a). Esta idea de autonomía en Ecuador queda en entredicho por autores como Waisbord (2013) que habla de "presidencialismo comunicacional" (p. 158) y por el fuerte paralelismo establecido entre los actores mediáticos y los políticos, creándose un contexto sociopolítico definido por un tipo de gobierno populista que ocasiona un tipo de periodismo propagandístico y controlador que genera obstáculos a la libertad de expresión y al acceso a la información, tanto a profesionales de los medios como a los ciudadanos. Flor (2013) en su análisis del diario El Universo acerca de los niveles de libertad de expresión en Ecuador, ratificando lo anteriormente expuesto por Waisbord (2013), muestra cómo los periodistas para evitar problemas, o ser llamados personalmente por el presidente del país, deben auto-censurar la información negativa que publican sobre el Gobierno.

Por lo que puede deducirse, como Amado y Amadeo (2013), que

el desempeño del periodismo en estos contextos se enfrenta a nuevos condicionamientos indirectos - publicidad oficial, asignación discrecional de licencias, administración arbitraria de sanciones, negación de acceso a la información pública, falta de acceso a los servidores públicos como fuentes de información, etc.-, más sutiles que la censura dictatorial pero no menos eficientes (p. 266).

7 Hay 6 categorías de perfiles ocupacionales en Ecuador: 1) Presentador locutor para radio y/o televisión; 2) Trabajador de piso de televisión; 3) Fotógrafo de medios y multimedia; 4) Coordinador técnico de programación para medios comunitarios; 5) Camarógrafo de noticias y 6) Productor de contenidos para medios comunitarios (cordicom, 2014).

8 Instituto Nacional de Normalización de Chile. Recuperado de http://www.inn.cl/pdfs/

9 Secretaría Nacional de Educación Superior, Ciencia, Tecnología e Innovación. Recuperado de http://www. educacionsuperior.gob.ec/

10 Servicio Ecuatoriano de Capacitación Profesional. Recuperado de http://www.secap.gob.ec/

11 Secretaría Técnica de Capacitación y Formación Profesional. Recuperado de http://www.secretariacapacitacion.gob.ec/ 


\section{Los factores determinantes de la autonomía profesional de los periodistas}

La profesión periodística está anclada a las bases estructurales que forman la sociedad de un país. Esta relación establece el grado de libertad y el nivel de autonomía profesional que definen y establecen los principios de su cultura periodística. En Ecuador, al igual que en el resto de países, existen predictores de la autonomía profesional. Reich y Hanitzsch (2013) hablan de 1) los límites objetivos de autonomía y 2) la percepción de autonomía de los periodistas con base en las influencias procedentes del contexto.

\section{Los niveles objetivos de autonomía profesional de los periodistas}

La autonomía a nivel individual, según Sjøvaag (2013), está relacionada con la libertad de cada individuo respecto al Estado, el control de los otros y la inmunidad del ejercicio arbitrario de la autoridad. O como dirían Glasser y Gunther (2005), todo lo referido a la "ausencia de condiciones"(p. 385). Según Sjøvaag (2013), la autonomía individual es una forma de autodirección de la libertad y de independencia moral respecto a los demás, que es el producto de la racionalidad y permite a la persona legislar por sí misma las leyes morales que la unen a todos. Este nivel individual de autonomía está indivisiblemente unido al nivel de autonomía institucional y al contexto socio-político y económico en el que se encuentra el periodista.

Respecto a la autonomía a nivel institucional, el periodismo es practicado dentro de unos límites impuestos por la institución y la autonomía profesional es negociada dentro del contexto institucional (Sjøvaag, 2013). Según Reich y Hanitzsch (2013), la autonomía profesional no es algo monolítico, sino que incorpora dos importantes facetas: la primera es la que relata la capacidad de acción que los periodistas tienen dentro de sus rutinas operacionales periodísticas - la autonomía se manifiesta en cómo los periodistas son libres de decidir sus historias, su edición y su cobertura, así como la elección de las fuentes informativas, los ángulos de análisis y las perspectivas-; la segunda se refiere a los aspectos vinculados directamente a la relación de los periodistas y las estructuras de ejecución y decisión dentro de la jerarquía editorial -hasta qué punto los periodistas pueden tomar parte en las decisiones que afectan a su trabajo y a los procesos de reportaje o edición-. De modo que a partir de estos autores, se puede hablar de tres niveles dentro de la autonomía institucional: 1) un nivel estructural formado por la estructura jerárquica formal, la estructura física del medio de comunicación y la propiedad de este; 2) un nivel organizacional basado en los factores organizacionales, los procesos, la posibilidad del periodista de interferir o intervenir en su actividad profesional y el producto final y 3) un nivel relacional centrado en el contexto institucional, donde la autonomía está asociada al nivel de independencia de otras instituciones sociopolíticas, principalmente el Estado y el mercado (Örnebring, 2013, p. 39; Sjøvaag, 2013, p. 156).

Finalmente, la autonomía a nivel de sistemas (entorno) está determinada por el sistema mediático, resultante de las condiciones políticas, económicas y jurídicas en el área de comunicación, y el contexto social, definido por los sistemas político y económico (Hallin \& Mancini, 2004; Hanitzsch, 2009; Oller \& Meier, 2012; Chavero \& Oller, 2014). De modo que los factores que determinan el resultado final del trabajo de los periodistas van del "nivel macro como la estructura social y política, al nivel micro formado por el trasfondo y la experiencia de los propios periodistas" (Sjøvaag, 2013, p. 158). 


\section{La percepción de autonomía profesional de los periodistas}

El concepto de autonomía se plantea como la idea paradigmática forjada en los esquemas formales que estructuran la profesionalización periodística. Bajo esta idea ejemplificadora de autonomía, asociada a su carácter epistemológico, este estudio deriva hacia la noción de 'percepción de autonomía profesional' como la capacidad que los periodistas poseen para percibir, decodificar, comprender y actuar a partir de las influencias procedentes del entorno (contexto). La diferenciación entre 'percepción de autonomía profesional', las 'influencias' y los 'límites objetivos', planteada por autores como Reich y Hanitzsch (2013), Mellado y Humanes (2012) o Sjøvaag (2013), lleva a plantear el concepto de 'percepción' bajo un criterio eminentemente pragmático, en el que se tiene en cuenta la experiencia personal de cada periodista a través de la cual comprende y percibe su entorno profesional, y que varía debido a factores espacio-temporales.

La percepción de la autonomía profesional de los periodistas ha sido evaluada y analizada desde las últimas décadas del siglo xx. Diversos estudios han sido presentados con el objetivo de trazar y sistematizar ciertos predictores de la autonomía profesional. Johnstone et al. (1976) encontraron que los periodistas proceden fundamentalmente de los grupos de las culturas predominantes en la sociedad y que los periodistas estadounidenses más jóvenes, con formación universitaria mantenían un mayor nivel de compromiso con valores asociados a la profesionalización del periodismo como la autonomía.

En la misma línea, Liebler (1994), Weaver et al. (2007) y Sjøvaag (2013) afirman que el tamaño y el tipo de la organización y el sistema de medios, el género, así como la raza y el ritmo de la información, son predictores significativos de la percepción de autonomía de los periodistas. Además, la posición jerárquica dentro de las redacciones determina la autonomía (Cook, 1998; Mellado \& Humanes, 2012; Ryfe, 2009; Willnat \& Weaver, 2003), donde los periodistas con más experiencia perciben una mayor independencia en su trabajo (Sigelman, 1973). De modo que la capacidad de los periodistas de intervenir e interferir en los procesos de toma de decisiones dentro de los medios de comunicación está directamente conectada a su percepción del nivel de autonomía profesional. Según Weaver et al. (2007), los periodistas consideran que pueden controlar sus propios procesos de trabajo cuando es mayor su sensación de interacción con el contexto organizacional. Al respecto, Hanitzsch et al. (2010) detectaron que las influencias organizacionales, profesionales y procedimentales eran percibidas como los límites más poderosos dentro del trabajo de los periodistas. Y que a mayor nivel de burocratización y especialización, menor control del producto final por parte de estos (Johnstone et al., 1976).

Mellado y Humanes (2012) afirman que las investigaciones (McChesney, 2003; Benson \& Neveu, 2005; Duval, 2005) que han mantenido el foco de estudio en las características que estructuran el campo periodístico con base en las fuerzas conflictivas, la autonomía profesional es mayor o menor dependiendo de si el capital simbólico del periodismo domina sobre las fuerzas comerciales. Al respecto existen dos posturas. En primer lugar, Weaver y Wilhoit $(1991 ; 1996 ; 2007)$ sugieren que las principales restricciones a la autonomía de los periodistas en EE.uu. proceden de las presiones impuestas por el mercado y que, según Duval (2005), los niveles de autonomía son inferiores en las organizaciones de medios privados y en las que dependen directamente de los ingresos procedentes de la publicidad. Por ejemplo, la exigencia de incluir publicidad en los medios de comunicación determina el contenido periodístico y la libertad editorial (McChesney, 2003). 
En segundo lugar, estudios recientes muestran que, aunque el contexto político y económico son las principales fuerzas que determinan las culturas periodísticas y los sistemas mediáticos (Hanitzsch \& Mellado, 2011, pp. 416-417), las influencias económicas y políticas ya no aparecen como fuerzas externas directas sobre los periodistas, sino como aspectos naturales del trabajo y, por lo tanto, como un factor insignificante a la hora de explicar la percepción de autonomía profesional que los periodistas poseen (Hanitzsch \& Mellado, 2011; Mellado \& Humanes, 2012; Reich \& Hanitzsch, 2013) al ser presiones percibidas de forma indirecta (McChesney, 2003).

A pesar de que los niveles de autonomía percibidos por los periodistas de todo el mundo mantienen un constante decrecimiento, las investigaciones en periodismo sitúan a la autonomía como un aspecto fundamental para los periodistas (Weaver et al., 2007) y un predictor de la satisfacción profesional (Demers, 1994; Stamm \& Underwood, 1993). "Altos niveles de autonomía profesional entre los periodistas se corresponden con altos niveles de libertad de prensa y bajos niveles de intervencionismo estatal en los medios de comunicación" (Reich \& Hanitzsch, 2013, p. 150). De forma que "los periodistas que trabajan en los países del oeste o en "democracias completas" perciben más autonomía que sus colegas en regímenes autoritarios o híbridos” (Reich \& Hanitzsch, 2013, p. 149).

\section{Niveles de influencias en la autonomía profesional de los periodistas}

En un sentido estricto, el juicio reflexivo es un atributo de la autonomía, pero como profesionales, los periodistas piensan sobre ellos mismos como actores autónomos dentro de unas normas de contexto, rutinas y tradición que envuelven los hábitos tanto como la introspección (McDevitt, 2003), por lo que las investigaciones deben tener en cuenta "la relación dialéctica entre los periodistas y sus instituciones, así como la idea del periodista como intermediario cultural” (Mellor, 2009, p. 309), sin perder de vista los estudios que superan la idea de mediación (Mazzoleni \& Schulz, 1999).

Por lo tanto, aunque el objeto del estudio sean los periodistas a nivel individual o como actores, la comprensión total de sus acciones queda determinada por los factores de influencia que los envuelven dentro de un contexto (Oller \& Chavero, 2015b). La percepción que los periodistas tienen de autonomía depende de las influencias que parten de las rutinas profesionales, la organización de los medios y del contexto en el que el periodista trabaja (Shoemaker \& Reese, 1991). La autonomía, como otros fenómenos sociales, es esencialmente un constructo subjetivo que principalmente existe en términos relativos (Reich \& Hanitzsch, 2013). Por lo que la naturaleza de esta no reside de forma autónoma en el individuo ni en la percepción aislada de este, sino que requiere de la contextualización y evaluación de todas las influencias internas y externas que afectan al trabajo periodístico y que determinan finalmente la idea de autonomía a nivel conceptual y empírico (Mellado \& Humanes, 2012).

La naturaleza multinivel de las influencias percibidas respecto a la autonomía refleja, esencialmente, una distinción epistemológica y estructural. La distinción epistemológica está basada en la diferenciación entre la percepción que tiene el periodista de las influencias que recibe sobre su autonomía periodística y los límites objetivos de esta. Aunque los límites objetivos de la autonomía pueden ser medidos dentro de un "mundo real", el significado final de autonomía reside en la percepción que el periodista tiene como individuo (Reich \& Hanitzsch, 2013).

Un estudio reciente de Oller y Meier (2012) desestructura el contexto en tres niveles de análisis superpuestos que establecen los tipos de influencias, su procedencia y su relación con la percepción de autonomía profesional que 
poseen los periodistas ${ }^{12}$. En la capa más externa, llamada nivel de sistemas, se encuentran los sistemas que estructuran el mercado, la economía, la política, la cultura y la sociedad que, como afirman autores como Weaver et al. (1996), McQuail (2006) o Reich y Hanitzsch (2013), proyectan influencias como la censura, las prohibiciones, las interferencias, la intimidación, las expectativas de beneficio o las consideraciones publicitarias. Hallin y Mancini (2004) sitúan en este nivel el desarrollo del mercado de los medios de comunicación, la relación y la cercanía con el poder político, el nivel de profesionalización -a partir de la autonomía de los periodistas, las normas profesionales y el valor dado al interés público-y el papel del Estado respecto a la libertad profesional de los periodistas.

La capa intermedia, formada por el nivel de institución, analiza la "estructura organizacional y las áreas de competencia" (Esser, 1998, p. 27) dentro de las redacciones de los medios. Estas determinan los factores de influencia procedentes de los perfiles de las actividades, el flujo de trabajo editorial, el control de la redacción, los mecanismos sociales (Esser, 1998) o la línea editorial del medio (Marr et al., 2001) que definen el trabajo de los periodistas. De forma que, como afirman Reich y Hanitzsch (2013), "el ambiente de la redacción es un fuerte condicionante del trabajo periodístico [...], provocando unas fuerzas que actúan como limitantes en la percepción profesional de autonomía de los periodistas" (p. 137).

La capa interior, denominada nivel de actor, detalla minuciosamente los intereses y métodos de trabajo en la redacción a nivel individual, quedando determinada por la formación, la experiencia profesional, la edad y el sexo del periodista (Johnstone et al., 1976). Según Reich y Hanitzsch (2013), las influencias proceden de los grupos de referencia profesionales - los colegas de otros medios, las audiencias y los procesos organizativos-y personales -los amigos, los conocidos y la familia-. En este nivel se encuentran los "factores con un fuerte carácter sociodemográfico, [...] muy valiosos a la hora de desarrollar el estudio empírico de una investigación” (Oller \& Meier, 2012, p. 147).

La investigación que se presenta en esta artículo involucra problemas que relacionan las percepciones de autonomía profesional de los individuos y la sociedad, aspecto que requiere de un análisis multinivel de las influencias contextuales. Por ello, la desestrucutración contextual permite establecer una jerarquía de influencias que "facilita la clasificación sistemática y la interpretación del material empírico" (Esser, 1998, p. 21).

\section{Metodología}

El proyecto CPE se basa en un método cualitativo, por lo que el trabajo de campo está acompañado de una exhaustiva investigación teórica previa que sirve como referencia, tal y como recomiendan autores como Mayring (2002) y Oller y Meier (2012). La muestra seleccionada consta de un total de 31 periodistas ecuatorianos (tabla 1) en activo con responsabilidad editorial dentro de la jerarquía de cada medio: redactores, coordinadores de sección, editores y jefes de redacción. Estos periodistas pertenecen a 6 medios de comunicación audiovisuales y escritos de propiedad privada, pública y comunitaria denominados "medios de calidad" por la influencia que ejercen sobre otros

12 Lo hace en relación a seis dimensiones: 1) percepción de los roles profesionales, 2) la idea de objetividad, 3) la ideología ética, 4) la percepción de las influencias, 5) el nivel de autonomía y 6) la confianza en las instituciones. 


\section{ESTUDIOS}

medios y su mediación en la opinión pública y en el proceso de creación de agenda en base a los temas actuales (Jarren \& Vogel, 2008, p. 18).

Tabla 1: Parámetros básicos de la muestra del estudio Cultura Periodística de Ecuador (CPE)

\begin{tabular}{|c|c|}
\hline Parámetros básicos de los periodistas entrevistados & Ecuador \\
\hline Periodistas entrevistados & 31 \\
\hline Mujeres periodistas (\%) & 32 \\
\hline Hombres periodistas (\%) & 68 \\
\hline Edad (M) & 35 \\
\hline Grado universitario en comunicación (\%) & 81 \\
\hline Años de experiencia como periodistas (M) & 11,1 \\
\hline Años de trabajo en el medio actual (M) & 6,3 \\
\hline \multicolumn{2}{|l|}{ Datos sociodemográficos } \\
\hline Autoubicación ideológica (\%) & $\begin{array}{l}\text { Izquierda o Centro-Izquierda: } 41,9 \\
\text { Centro: } 35,5 \\
\text { Derecha: } 6,5\end{array}$ \\
\hline Ocupación de un cargo político (\%) & $\begin{array}{l}\text { No: } 96,8 \\
\text { Sí: } 3,2\end{array}$ \\
\hline Interés en la política & $\begin{array}{l}\text { Bastante o mucho: } 45,2 \\
\text { Medio: } 29 \\
\text { Poco o nada: } 25,8\end{array}$ \\
\hline \multicolumn{2}{|l|}{ Datos de los medios de comunicación } \\
\hline Propiedad de los medios & $\begin{array}{l}\text { Públicos: } 41,9 \\
\text { Privados: } 51,6 \\
\text { Comunitarios: } 6,5\end{array}$ \\
\hline Tipo de medios & $\begin{array}{l}\text { Audiovisuales: } 58,1 \\
\text { Escritos: } 41,9\end{array}$ \\
\hline
\end{tabular}

Fuente: Elaboración propia.

Las entrevistas en profundidad se llevaron a cabo cara a cara durante el primer semestre de 2014 en las redacciones de cada uno de los medios analizados. El cuestionario ${ }^{13}$ utilizado estuvo compuesto principalmente por preguntas abiertas estructuradas - aunque en el análisis de algunas dimensiones se utilizaron preguntas cerradas - con base en 6 ejes temáticos (el rol profesional de los periodistas, el concepto de objetividad, la ética periodística, las principales influencias percibidas, el grado de autonomía y el nivel de confianza en las instituciones)

13 La fiabilidad y la validez de este cuestionario fue contrastada con estudios previos realizados en países como España y Suiza. 
más un apartado dedicado a las preguntas socio-psicográficas. Los datos obtenidos fueron estudiados a partir de un análisis de contenido basado en la minería de datos - texto oral-. Para ello se utilizó el software Antconc que facilitó la creación de categorías a partir de la ocurrencia y la concordancia de las unidades léxicas - palabras y segmentos-que formaban los modelos mentales comunes.

La selección de la muestra se realizó buscando la mayor representatividad de los periodistas y de los medios de comunicación en Ecuador. Para ello, se efectuó la selección de la muestra a partir de un muestreo por cuotas para obtener una representación lo más significativa posible en cuanto a género, cargo y naturaleza del medio ${ }^{14}$. Aunque se cuenta con una muestra no representativa, autores como Wyss (2002) estiman que en un estudio cualitativo el total de entrevistas presentadas en este estudio es un número aceptable y que puede tomarse como referencia.

Los resultados se analizaron de forma contextualizada con base en los niveles de influencia percibidos por los periodistas (Oller \& Meier, 2012).

\section{Resultados}

Los resultados muestran que las tres cuartas partes $(74,2 \%)$ de los periodistas entrevistados afirma que tiene total libertad en el trabajo desarrollado dentro de su redacción: "Sí, por supuesto. Cien por ciento" (6);

Sí, son muy abiertos aquí en el canal [televisión] para ese tipo de cosas, si uno cree que uno va a afectar con la noticia a una persona o se afecta a sí mismo o afecta a la empresa tomamos una decisión en grupo. Generalmente es así, si a los directores se les ocurre un tema, lo tienen en cuenta a uno, que desde su experiencia como reportero de la calle les dice que va a ser difícil o complicado, que no es verdad o que es un rumor que hay; sí hay libertad en ese sentido (17).

En esta misma línea, el 22,6\% considera que su libertad está condicionada por la línea editorial del medio o sus superiores, lo cual supone reconocer cierto grado de autocensura marcado por la línea editorial del medio al que pertenece: "Sí, no he tenido ninguna limitación cuando uno conoce la línea editorial en la que más o menos se desenvuelve el medio" (18). "Sí, tengo la libertad de escoger los temas que para mí son importantes, y si no, estoy abierta a las opiniones o sugerencias de jefes o compañeros de trabajo" (14); "Bastante, no la que quisiera. [...] En gran medida sí puedo tomar parte en las decisiones que afectan a mi trabajo" (7). De modo que el 96,8\% de los periodistas entrevistados considera que disfruta de libertad - total o parcial- respecto a sus superiores y en el trabajo que realiza en su medio de comunicación.

14 Esta característica marca los propios límites de la investigación. 
Gráfico 1: ¿Considera que tiene mucha libertad en el trabajo que realiza en su medio de comunicación?

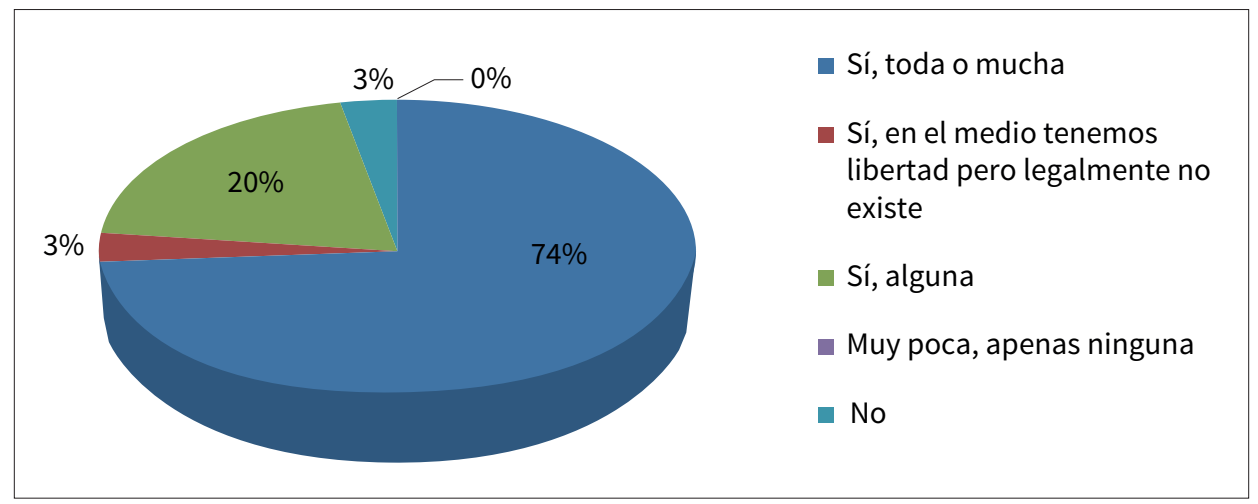

Fuente: Elaboración propia.

Respecto a la posibilidad de los periodistas de tomar parte en las decisiones que afectan a su trabajo, el 83,9\% afirma que sí lo puede hacer: "Sí puedo tomar decisiones: discutir con mi jefe o con mis compañeros directos" (14); "Sí, ciento por ciento en todos los casos desde el planteamiento de propuestas periodísticas hasta la realización" (15); "Sí, en el trabajo periodístico" (25).

El resto de entrevistados, a pesar de no tener una participación directa en la toma de decisiones, ratifica que tiene algún tipo de participación (6,5\%): "La mayor parte de veces, sí" (1); "a veces sí, a veces sí se pude hacer eso, claro inconscientemente uno lo hace, decisiones que afectan al trabajo" (20); mientras que un 9,7\% de los entrevistados afirma que a pesar de no participar en la toma de decisiones de manera directa, se les escucha o se tiene en cuenta su opinión: "Tomar parte en las decisiones no, pero sí puedo dar mi punto de vista, creo que mi jefe sí da valor a lo que yo pienso" (9).

Gráfico 2: ¿Puede tomar parte en las decisiones que afectan a su trabajo?

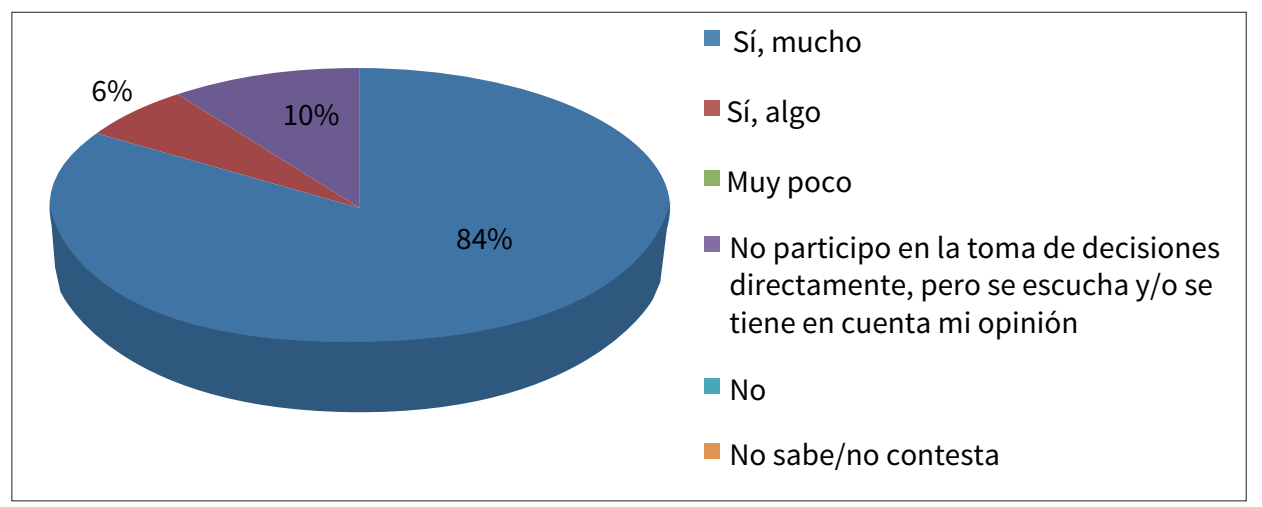

Fuente: Elaboración propia. 
Los periodistas entrevistados consideran que los límites más significativos a su libertad de expresión parten de la nueva legislación en materia de comunicación. Algo más de una cuarta parte $(27,4 \%)$ destaca la influencia de la toc de 2013: "Las leyes que frenan la actividad del periodista” (24); “[...] Diría que la Ley de Comunicación” (13). Algunos de los entrevistados definieron esta ley como punitiva, capaz de ocasionar el efecto de autocensura en los periodistas: "Límites en la libertad de expresión sí, hay autocensura por la Ley de Comunicación, nos cuidamos mucho en decir o sacar algo" (16);

Me parece que desde la normativa que se ha instaurado acá en el país, por la nueva Ley de Comunicación [...], porque creemos que ahora los periodistas de este medio sí tienen algo de temor a sanciones, a represalias, entonces, lo que se genera [...] es la autocensura (12).

Yo creo que hay una especie de judicialización del periodismo, ese es el limitante más importante, eso es lo que hace que los temas sean más tibios de lo que deberían ser. Este rato hay una locura por los juicios, a todo el mundo le gusta poner juicios (18).

El 17,7\% de los periodistas entrevistados considera que la ética profesional, el respeto a las personas y sus derechos son los principales límites a la libertad de expresión: "¿Los límites más significativos a mi libertad? Los derechos de la otra persona" (4);

Los valores, esos valores que son muy fundamentales, ese es un criterio importante; sí, es como que en eso nos movemos aquí, entonces vemos cómo le puede afectar a alguien tal cosa, cómo le afectaría el valor de la libertad, el respeto" (25).

El tercer factor considerado por los entrevistados como el más importante respecto a la limitación de su libertad de expresión se encuentra en el nivel de institución -como ya se confirmó en la primera pregunta-, el 16,1\% afirma que estos límites proceden de los intereses o presiones del medio o de la empresa: "Yo pienso que límites no hay como periodista, siempre y cuando uno se rija a la línea editorial del medio" (14); "Creo que la línea editorial realmente sí es una limitante para la libertad de expresión en cualquier medio y eso, la verdad, porque igual la línea editorial está basada por los intereses del medio" (31).

Por otro lado, casi un cuarto de los entrevistados $(22,6 \%)$ considera que no existen límites a la libertad de expresión en su medio de comunicación o que el periodista es quien marca sus propios límites: "Creo que no hay una limitación, la limitación se le hace uno mismo [...] (20); "Creo que tienen que ver con la autocensura, a veces uno mismo se pone los candados y el tapabocas" (23);

Yo creo que, dentro de mi ideología política, no tendría límites, porque lo que yo quiero es que se vea el trabajo del Gobierno, y si es que yo presento cualquier reportaje con esa perspectiva, voy a tener total apertura de hacerlo (19).

Finalmente, el 16,2\% de los periodistas relaciona su libertad de expresión a los intereses y acciones del Gobierno, el Estado o la Administración Pública y a la falta de acceso a la información: "Cierta no sé si llamarle persecución de la Superintendencia que me parecen que son un poco autoritarias" (28);

Los límites que tengo como periodista, como la libertad de expresión, creo que es más el acceso a la información [...], porque a veces uno se formula y quiere cumplirlos y hace los pedidos, pero nunca tenemos respuesta; en ocasiones no se tiene [...] (27);

Tener un grado de dependencia directa o indirecta de quienes hacen la administración pública, porque nosotros recibimos recursos del Estado y eso puede ser malinterpretado por determinadas instituciones 
o quienes están en determinados puestos en las instituciones públicas, para creer que nosotros somos subalternos de ellos o debemos escribir lo que a ellos les parece (7).

Gráfico 3: ¿Cuáles considera que son los límites más significativos a su libertad de expresión como periodista?

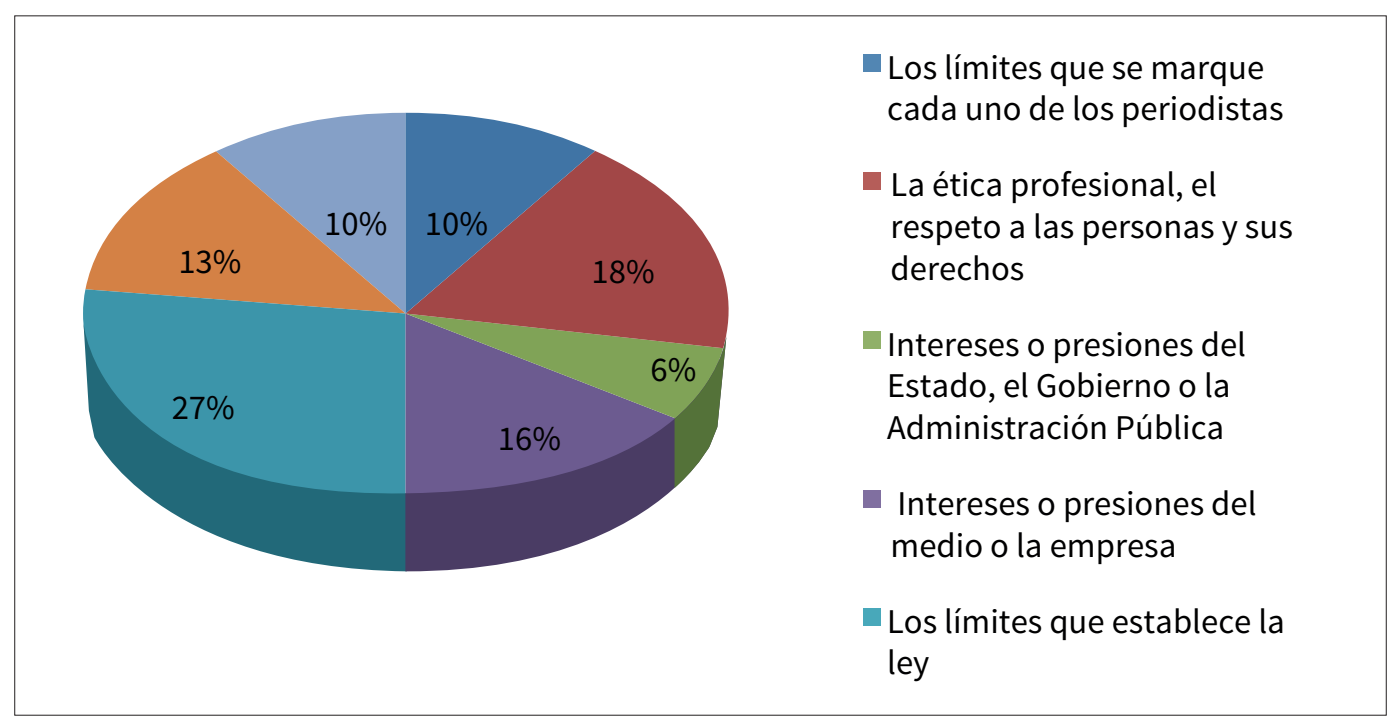

Fuente: Elaboración propia.

\section{Conclusiones}

Una de las características de la cultura periodística de Ecuador, según los resultados obtenidos, es la percepción de un alto nivel de autonomía por parte de los periodistas, gracias a la cual ejercen su actividad profesional con casi o total libertad. A este aspecto contribuye el hecho de que forman parte de las decisiones que afectan su trabajo, o al menos son escuchados por sus superiores. Estos resultados coinciden con los obtenidos por Mellado y Humanes (2012) y Skovsgaard (2013) en sus estudios sobre la percepción de la autonomía profesional de los periodistas en Chile ${ }^{15}$ y Dinamarca, contradiciendo la tendencia de otros estudios internacionales realizados en España por García de Cortázar y García de León (2000) y por Weaver et al. (2007) en EE.uu., donde los periodistas afirman poseer niveles moderados de autonomía y el de Reich y Hanitzsch (2013), que determina que los periodistas en

15 El estudio llevado a cabo en Chile por Sapiezynska, Lagos y Cabalin (2013) rebate estos resultados al afirmar que en los medios de comunicación de este país existen unos niveles de restricción considerables a la libertad de prensa. 
países con "democracias completas" perciben un mayor nivel de autonomía que los que trabajan en países con regímenes híbridos.

Estos resultados contradictorios cuestionan la idea de autonomía como concepto global y estandarizado dentro del periodismo, que muestra una idea dinámica, heterogénea e híbrida adaptada a los diferentes contextos y culturas periodísticas. Aunque la autonomía pueda verse como parte integral del modelo normativo del periodismo occidental (Sjøvaag, 2013), que creció a partir del relativo monopolio del mercado norteamericano a finales del siglo xix y que se expandió al resto del mundo (Nerone, 2013), en términos prácticos, tal y como exponen los resultados de este estudio y demostró Ryfe (2009), es algo que es negociado a nivel local a través de la interacción entre los dueños, los editores y los periodistas. De modo que se afianza la idea de una cultura periodística latinoamericana que, aunque con marcados rasgos globales, es heterogénea, diversa y con una idiosincrasia y unas características propias que difieren de los estándares periodísticos occidentales y, por lo tanto, definen la idea y la percepción del nivel de autonomía de los periodistas que trabajan en ella. Así lo mostró en las últimas décadas del siglo xx Ruótolo (1987), quien concluyó que las diferencias encontradas en su estudio -en la que la autonomía profesional fue una de las cuatro dimensiones de análisis- en tres países latinoamericanos - Brasil, Colombia y Costa Rica - claramente indicaban que la orientación profesional de los periodistas en América Latina varía ampliamente de un país a otro.

El estudio muestra la diferencia existente entre cómo los periodistas perciben su autonomía y los niveles objetivos de esta. Aunque los entrevistados asocian, en la misma línea de los resultados obtenidos por Örnebring (2013), el concepto de autonomía con los de legitimidad y autoridad profesional, aceptan que su trabajo está relacionado directamente con las estructuras de ejecución, organización y posicionamiento (línea editorial) de los medios de comunicación y la configuración socio-política y económica del país.

A pesar de los altos niveles de autonomía percibidos por los entrevistados (para esta investigación), cuando se les pregunta específicamente por la existencia de determinadas influencias que de una forma u otra limitan su libertad de expresión como periodista, el $87 \%$ de los entrevistados responde de forma afirmativa. Entre los principales límites que encuentran son: 1) los establecidos por la ley y la actual legislación en comunicación; 2) los estipulados por la ética profesional, el respeto a las personas y sus derechos y 3 ) los establecidos por las presiones y los intereses de los medios de comunicación.

En primer lugar, más de un tercio de los entrevistados (33,9\%) plantea como principal limitación la legislación vigente, específicamente la Loc y los intereses o presiones del Estado, del Gobierno o la Administración Pública. El temor a ser sancionados por los órganos reguladores tomando como base la Loc ocasiona, por un lado, la autocensura de los periodistas ${ }^{16}$ y, por otro, la disminución del énfasis en la investigación y el análisis, exponiendo una información que podría ser definida como "tibia"17.

Este resultado muestra las influencias procedentes del nivel de sistemas según determinan Oller y Meier (2012) y el nivel relacional expuesto por Örnebring (2013), Sjøvaag (2013) y Reich y Hanitzsch (2013). Este rol inter-

16 Tal y como afirma Flor (2013) en su estudio sobre la percepción de libertad de expresión en los periodistas del diario El Universo.

17 Tal y como afirman Amado y Amadeo (2013) al mostrar que el desempeño del periodismo en estos contextos se enfrenta a nuevos condicionamientos indirectos. 
vencionista jugado por el Gobierno en la actividad periodística como regulador y financiador de los medios de comunicación sitúa a Ecuador entre los países que, tal y como determinan Reich y Hanitzsch (2013), poseen un sistema mediático sometido a una estricta regulación y en el que los periodistas poseen una autonomía profesional limitada; corroborando lo dicho por Hallin y Mancini (2004) previamente al afirmar que los periodistas que trabajan en sistemas mediáticos definidos por el fuerte paralelismo político entre medios y Estado gozan de menores niveles de autonomía.

De modo que, el posicionamiento que tradicionalmente han mantenido los medios de comunicación en Ecuador hasta la llegada del presidente Rafael Correa - no puede olvidarse que el 100\% era de propiedad privada- está evolucionando hacia un punto donde el paralelismo con el Estado es cada vez mayor. Prueba de ello son los resultados obtenidos por Wilke (1998) en el que la opción de "apoyar al Gobierno en el desarrollo de sus programas" (p. 441) obtuvo el menor consenso entre los periodistas encuestados. Si se comparan los resultados obtenidos por Wilke en la década de los noventa del siglo pasado y los de este estudio -en el que se demuestra la fuerte influencia de la nueva legislación y del Gobierno en la percepción de libertad de expresión en los periodistas entrevistados-, queda demostrada la evolución hacia una cultura periodística que abandona el concepto neoliberal que imperó en las últimas décadas del siglo xx y la primera del siglo xxı y que se acoge a un modelo sociodemocrático en el que el control y la influencia del Gobierno es cada vez mayor.

En segundo lugar, los periodistas entrevistados estiman como principales limitantes a su libertad de expresión las barreras autoimpuestas por las normas éticas, los valores y los criterios recogidos dentro de los códigos deontológicos de los medios. Los resultados confirman lo expuesto, en primer lugar, por Reese (1999) al afirmar que el trabajo de los periodistas en las redacciones se basaba en la adhesión a un código de conducta y, en segundo lugar, por Berkowitz y Limor (2003) al mostrar que las conductas éticas, a pesar de estar fuertemente determinadas por los distintos niveles de influencia - nivel de actor, nivel organizacional y nivel de sistemas - actúan como factores determinantes de la autonomía tal y como se transmite en la formación periodística universitaria. Estas normas educativas estructuran los ideales y las ideologías profesionales en torno a las conductas éticas y la independencia periodística - autonomía-. De forma que la formación universitaria de los periodistas entrevistados - casi toda la muestra ha estudiado Comunicación Social- influye en su percepción de autonomía y les lleva a concretar sus prácticas profesionales de una manera determinada, confirmando lo expuesto por Fröhlich y Holtz-Bacha (2003).

En tercer lugar, los entrevistados se refieren a las presiones e intereses de los medios de comunicación. Estos límites a la libertad de expresión de los periodistas entrevistados quedarían definidos con base en las influencias recibidas procedentes de los niveles de institución y de actor (Oller \& Meier, 2012), concretamente por la estructura y la organización de los medios que determinan los intereses, las competencias y los métodos de trabajo en la redacción. Este resultado corroboraría, al menos parcialmente, los argumentos mostrados por autores como McChesney (2003), Duval (2005) y Weaver et al. (2007) sobre la influencia de la concentración, la propiedad y la comercialización de noticias, así como las expectativas de negocio de los medios en la autonomía profesional.

El estudio muestra la realidad mediática existente hoy en día en Ecuador, en la que el $90 \%$ de los medios son de propiedad privada -y por lo tanto, con una mayor orientación de mercado en busca de beneficios económicos y un mejor posicionamiento- y en el que a partir de la llegada al poder del presidente Rafael Correa (2007) se han ido forjando políticas públicas a favor de una regulación de la actividad periodística en el país y de una reestructuración del sistema mediático en favor del denominado "Bien Común". Esta propuesta gubernamental se 
ha consolidado a partir de la aprobación la Ley Orgánica de Comunicación en 2013 y a la creación de organismos de regulación COMO la SUPERCOM y el CORDICOM. El efecto de estas estrategias está llevando a los periodistas a percibir esta propuesta de regulación externa a la profesión como unos de los principales limitantes a su autonomía profesional.

Evidencia que apesar de la heterogeneidad de la cultura periodística de América Latina, existen puntos en común entre sus países (Oller \& Barredo, 2013). En el caso de Ecuador, los periodistas mantienen un conflicto profesional procedente de la relación entre 1) el fuerte control del sistema mediático, tal y como afirmó Ruótolo (1987), 2) la influencia de la concentración de los medios de comunicación, afirmado por Weaver et al. (2007) y 3) la percepción de independencia y autonomía profesional de los periodistas.

Finalmente, los periodistas perciben un alto nivel de autonomía profesional y, por lo tanto, se encuentran satisfechos con su situación. A pesar de ello, la mayor parte reconoce presiones e influencias procedentes del contexto que limitan o determinan su libertad de expresión. La razón que explicaría estos resultados podría centrarse, en primer lugar, en la relación que los periodistas realizan entre los conceptos de ética, autonomía y profesionalismo y, en segundo lugar, en la no existencia de una cultura periodística ecuatoriana madura sustentada en una profesionalización sólida del periodismo.

Estos resultados muestran el camino a seguir en los estudios realizados en países como Ecuador, englobados dentro de las denominadas culturas periodísticas intermedias, donde su idiosincrasia les lleva a tener un modelo periodístico propio. De modo que se invita a los investigadores a continuar trabajando los temas de culturas periodísticas bajo una perspectiva contextualizadora.

\section{Bibliografía}

1. Abad, G. (2013). Medios, periodismo y responsabilidad social: en busca de políticas públicas en el Ecuador. Chasqui, (122), 13-22. Recuperado de http://repositorio.ciespal.org:8080/bitstream/123456789/1316/1/ CIESPAL_Chasqui_Medios,_periodismo_y_responsabilidad_social_en_busca_de_pol\%C3\%ADticas_p\%C3\%BAblicas_en_el_Ecuador.pdf

2. Aldridge, M., \& Evetts, J. (2003). Rethinking the concept of professionalism: the case of journalism. British Journal of Sociology, 54(4), 547-564. Recuperado de http://onlinelibrary.wiley.com/doi/10.1111/j.14684446.2003.00547.x/pdf

3. Amado, A., \& Amadeo, B. (2014). Periodismo, ética y propaganda en la Argentina democrática. Estudos em Jornalismo e Mídia, 11(1), 266-276. Recuperado de https://www.academia.edu/7632081/Periodismo_ \%C3\%A9tica_y_propaganda_en_la_Argentina_democr\%C3\%A1tica

4. Aznar, H. (2011). Comunicación responsable. La autorregulación de los medios (2 ${ }^{\text {da }}$ edición). Barcelona: Ariel.

5. Beam, R. (1990). Journalism professionalism as an organizational-level concept. Journalism Monographs, (121), 1-43.

6. Benson, R., \& Neveu, E. (2005). Bourdieu and the journalistic field. Cambridge, UK: Polity. 
7. Berkowitz, D., \& Limor, Y. (2003). Professional confidence and situational ethics: Assessing the Social-Professional dialectic in Journalistic Ethics Decesions. J\&MC Quarterly, 80(4), 783-801. Recuperado de http:// jmq.sagepub.com/content/80/4/783.full.pdf+html

8. Carr-Saunders, A. M., \& Wilson, P. (1933). The professions. Oxford University Press.

9. Chavero, P., \& Oller, M. (2014). La comunicación en Ecuador ¿derecho o servicio público? Revista Culturas Jurídicas, 1(1). Recuperado de http://www.culturasjuridicas.uff.br/index.php/rcj/article/view/63

10. Chavero, P., González, J.J., Bouza, F., Castromil, A., \& Rodríguez, R. (2013). La mediatización de la agenda política: la discusión del pacto social como conflicto de agendas. 2009-2011. Revista Latina de Comunicación Social, (68). Recuperado de http://www.revistalatinacs.org/068/paper/994_Complutense/28_Palmira.html

11. Checa-Godoy, A. (2012). The banking sector and media ownership: the case of Ecuador. Revista Latina de Comunicación Social, (67), 125-147. Recuperado de http://www.revistalatinacs.org/067/art/950_ Sevilla/06_ChecaEN.html

12. Consejo de Regulación y Desarrollo de la Información y Comunicación, CORDICOM (2014). Resultados encuesta de profesionalización. Recuperado de http://www.cordicom.gob.ec/resultados-de-la-encuesta-nacional-de-profesionalizacion-a-los-trabajadores-de-la-comunicacion/

13. Cook, T. E. (1998). Governing with the news: The news media as a political institution. Chicago: University of Chicago Press.

14. Demers, D. P. (1994). Effect of organizational size on job satisfaction of top editors at U.S. dailies. Journalism Quarterly, 71(4), 914-925. Recuperado de http://jmq.sagepub.com/content/71/4/914.abstract

15. Duval, J. (2005). Economic journalism in France. En R. Benson \& E. Neveu (Eds.). Bourdieu and the journalistic field. Cambridge, UK: Polity.

16. Elliott, P. (1972). The sociology of the professions. London: Macmillan.

17. Esser, F. (1998). Die Kräfte hinter den Schlagzeilen. Englischer und deutscher Journalismus im Vergleich. Freiburg, München: Verlag Karl Albert.

18. Flor, M. J. (2013). Goberning Through Permanent Campaigning: Media Usage and Press Freedom in Ecuador. (Tesis de pregrado catalogada en University Libraries Lance y Elena Calvert, Universidad de Nevada, Las Vegas).

19. Fröhlich, R., \& Holtz-Bacha, C. (2003). Journalism education in Europe and North America. An international comparison. Cresskill, New Jersey: Hampton Press, Inc.

20. García de Cortázar, M., \& García de León, M. A. (2000). Profesionales del periodismo. Hombres y mujeres en los medios de comunicación. Madrid: cis.

21. Glasser, T. L., \& Gunther, M. (2005). The Legacy of Autonomy in American Journalism. En G. Overholser \& K. H. Jamieson (Eds.). The Press. Oxford University Press.

22. Hallin, D. C., \& Mancini, P. (2004). Comparing media systems: Three models of media and politics. Cambridge University Press.

23. Hanitzsch, T. (2007). Deconstructing journalism culture: Toward a universal theory. Mass Communication Theory, 17(4), 367-385. 
24. Hanitzsch, T., \& Mellado, C. (2011). What Shapes the News around the World? How Journalists in Eighteen Countries Perceive Influences on Their Work. The International Journal of Press/Politics, 16(3). Recuperado de http://hij.sagepub.com/content/early/2011/04/28/1940161211407334

25. Hanitzsch, T., Anikina, M., Berganza Conde, R., Cangoz, I., Coman, M., Hamada, B., ... Wang Yuen, K. (2010). Modeling perceived influences on journalism: evidence from a cross-national survey of journalists. Journalism \& Mass Communication Quarterly, 87(1), 7-24. Recuperado de http://eprints.qut.edu. au/68292/1/2010_-_Hanitzsch_et_al___JMCQ_-_Modeling_Perceived_Influences.pdf

26. Hervieu, B., \& Samson, E. (2010). El volcán mediático y su delicada regulación. Informe sobre Ecuador. París: Reporteros sin Fronteras.

27. Jarren, O., \& Vogel, M. (2008). Gesellschaftliche Selbstbeobachtung und Ko-Orientierung. Die Leitmedien der modernen Gesellschaft. En Gendolla, P., Ligensa, A., y Müller, D. (Eds.). Alte und neue Leitmedien. Bielefeld: Transcript Verlag.

28. Johnstone, J. W. C., Slawski, E. J., \& bowman, W. W. (1976). The news people: A sociological portrait of American journalists and their work. Urbana: University of Illinois Press.

29. Liebler, C. (1994). How race and gender affect journalists' autonomy. Newspaper Research Journal, 15(3), 122-130.

30. Löffelholz, M., \& Weaver, D. (2008). Global journalism research. Summing up and looking ahead. En Löffelholz, M. y Weaver, D. H. (Eds.). Global journalism research. Theories, methods, findings, future. Oxford: Blackwell Publishing.

31. Marr, M., Wyss, V., Blum, R., Bonfadelli, H., \& Beck, D. (2001). Journalisten in der Schweiz. Eigenschaften, Einstellungen, Einflusse. Konstanz: Publizistik Vierteljahreshefte fur K.

32. Mayring, P. (2002). Einführung in die qualitative Sozialforschung. Eine Anleitung zu qualitativem Denken, (5 $5^{\mathrm{a}}$ ed.). Weinheim, Basel: Beltz Verlag.

33. Mazzoleni, G., \& Schulz, W. (1999). 'Mediatization' of politics: a challenge for democracy? Political Communication, (16), 247-261. Recuperado de http://www.tandfonline.com/doi/pdf/10.1080/105846099198613?redirect=1\#.VplSfPI97IU

34. McChesney, R. W. (2003). The Problem of Journalism: A Political Economic Contribution to and Explanation of the Crisis in Contemporary US Journalism. Journalism Studies, 4(3), 299-329.

35. McDevitt, M. (2003). In Defense of Autonomy: A critique of the Public Journalism Critique. Journal of Communication, 53(1), 155-164.

36. McQuail, D. (1994). Normative theories of media performance, mass communication theory. An introduction. London: SAGE Publications.

37. McQuail, D. (2006). New horizons for communication theory in the new media age. En: Valdivia, A. (Ed.). A companion to media studies. Oxford: Blackwell Publishing.

38. Mellado, C., \& Humanes, M. L. (2012). Modeling perceived professional autonomy in Chilean journalism. Journalism, 1(19). Recuperado de http://jou.sagepub.com/content/13/8/985.abstract

39. Mellor, N. (2009). Strategies for autonomy. Arab journalists reflecting on their roles. Journalism Studies, 10(3), 307-321. Recuperado de http://www.tandfonline.com/doi/pdf/10.1080/14616700802636243 
40. Merrill, J. C. (1974). The imperative of freedom: A philosophy of journalistic autonomy. New York: Hastings House.

41. Murillo-Ruiz, C. (2011). El vía Crucis político y mediático de la protesta social en Ecuador. En: Rabinovich, E., Magrini, A. L., y Rincón, O. (Eds.). Vamos a portarnos mal. Protesta social y libertad de expresión en América Latina. Bogotá: Friedrich Ebert Stiftung.

42. Nerone, J. (2013). The historical roots of the normative model of journalism, Journalism, 14(4): 446-458. Recuperado de http://jou.sagepub.com/content/early/2012/10/24/1464884912464177

43. Oller, M., \& Barredo, D. (2013). Las culturas periodísticas intermedias. Estudios comparativos internacionales en periodismo. La Laguna (Tenerife): Sociedad Latina de Comunicación Social.

44. Oller, M., \& Chavero, P. (2015a). La profesionalización del periodismo y el profesionalismo de los periodistas en Ecuador. Prisma.com. Revista de Ciencias e Tecnologias de Informacao e Comunicacao, (25), 23-49. Recuperado de http://revistas.ua.pt/index.php/prismacom/article/view/3045/pdf_27

45. Oller, M. \& Chavero, P. (2015). La percepción de los factores de influencia de los periodistas dentro de la cultura periodística de Ecuador. Anuario Electrónico de Estudios en Comunicación Social "Disertaciones", 8, (1), 81-104. Doi: dx.doi.org/10.12804/disertaciones.01.2015.04

46. Oller, M., \& Meier, K. (2012). La cultura periodística de España y Suiza. Madrid: Fragua.

47. O'Neil, P. (2010). Essential of Comparative Politics. (3 ${ }^{\text {rd. }}$ Ed.). New York: W. W. Norton \& Company.

48. Örnebring, H. (2013). Anything you can do, I can do Better? Professional Journalists on Citizen Journalism in Six European Countries. International Communication Gazette, 75(1), 35-53. Recuperado de http://gaz. sagepub.com/content/75/1/35.full.pdf+html

49. Ortega, F., \& Humanes, M.L. (2001). Algo más que periodistas. Sociología de una profesión. Barcelona: Ariel.

50. Programa Internacional para el desarrollo de la Comunicación (2011). Análisis del Desarrollo Mediático en el Ecuador - 2011. Quito: Unesco.

51. Punín, M. I. (2011). Rafael Correa y la Prensa Ecuatoriana: una Relación de Intrigas y Odios. Razón y Palabra, (75). Recuperado de http://www.razonypalabra.org.mx/N/N75/varia_75/varia3parte/37_Punin_V75.pdf

52. Ramos, I. (2013). Trayectorias de democratización y desdemocratización de la comunicación en Ecuador. íconos. Revista de Ciencias Sociales, (45), 67-82. Recuperado de https://www.flacso.edu.ec/portal/index. php? module=MediaAttach \&func $=$ download\&fileid $=149$

53. Reese, S. D. (1999). The progressive potential of journalism education: Recasting the academic versus professional debate. Harvard International Journal of Press/Politics, (4), 70-94. Recuperado de http://hij. sagepub.com/content/4/4/70.abstract

54. Reetberg, A., \& Rincón, O. (2012). Medios, democracia y poder: una Mirada comparada desde Colombia, Ecuador, Venezuela y Argentina. (1 ${ }^{\text {era }}$ Ed.). Bogotá: Universidad de los Andes.

55. Reich, Z., \& Hanitzsch (2013). Determinants of Journalists' Professional Autonomy: Individual and National Level Factors Matter More Than Organizational Ones. Mass Communication and Society, 16(1), 133-156.

56. Reporteros sin Fronteras (2010). Informe Anual. La libertad de prensa en el mundo en 2010. Madrid: Reporteros sin Fronteras. 
57. Reyes, H. (2014). Activismo estatal y democratización social en Ecuador. Tensiones contrahegemónicas frente al poder mediático (2007-2013). Nueva Sociedad, (249), http://www.nuso.org/upload/articulos/4007_1.pdf

58. Ruótolo, A. C. (1987). Professional orientation among journalists in three Latin American countries. International Communication Gazette, (40), 131-142. Recuperado de https://www.researchgate.net/publication/245517789_Professional_orientation_among_journalists_in_three_Latin_American_countries

59. Ryfe, D. M. (2009). Broader and Deeper: A Study of Newsroom Culture in a Time of Change, Journalism, 10(2), 197-216. Recuperado de http://jou.sagepub.com/content/10/2/197.abstract

60. Sapiezynska, E., Lagos, C., \& Cabalin, C. (2013). Libertad de prensa bajo presión: niveles de restricción percibidos por periodistas chilenos y factores influyentes. Cuadernos.info, (32), 11-26. Recuperado de http://cuadernos.info/index.php/CDI/article/view/468/pdf

61. Schlesinger, P. (1978). Putting "Reality" together: BBC News. London: Methuen.

62. Shoemaker, P. J., \& Reese, S. (1991). Mediating the message. Theories of influences on mass media content. New York: Longman.

63. Sigelman, L. (1973). Reporting the News: An Organizational Analysis. The American Journal of Sociology, (79), 132-51. Recuperado de https://www.researchgate.net/publication/249171832_Reporting_the_News_ An_Organizational_Analysis

64. Sjøvaag, H. (2013). Journalistic Autonomy. Between Structure, Agency and Institution. Nordicom Review, (34), 155-166. Recuperado de http://www.nordicom.gu.se/sites/default/files/kapitel-pdf/12_sjovaag_0.pdf

65. Skovsgaard, M. (2013). Watchdogs on a leash? The impact of organizational constraints on journalists perceived professional autonomy and their relationship with superiors. Journalism, 15(3), 344-363. Recuperado de http://jou.sagepub.com/content/15/3/344.abstract

66. Stamm, K. \& Underwood, D. (1993). The Relationship of Job Satisfaction to Newsroom Policy Changes. Journalism Quarterly, (70), 528-541. Recuperado de http://jmq.sagepub.com/content/70/3/528.abstract

67. Waisbord, S. (2013). Vox Populista: Medios, periodismo, democracia. Buenos Aires: Gedisa.

68. Weaver, D. H., \& Wilhoit G. C. (1991). The American journalist. A portrait of U.S. news people and their work. Bloomington: Indiana University Press.

69. Weaver, D. H., \& Wilhoit G. C. (1996). The American journalist in the 1990s: U.S. news people at the end of an era. Mahwah. NJ: Erlbaum.

70. Weaver, D.; Randal A. B.; Brownlee, B. J.; Voakes, P. S., \& Wilhoit, G. C.. (2007). The american journalist in the 21st century. U.S. News people at the dawn of a new millennium. New Jersey: Lawrence Erlabaum Associates, Publishers.

71. Wilke, J. (1998). Journalists in Chile, Ecuador and Mexico. En D. H. Ewaver (Ed.). The Global Journalist, News, People around the World. New Jersey: Hampton Press, Inc.

72. Willnat, L. \& Weaver, D. (2006). Journalism and communication education in the United States. En K. Leung \& P. Lee (Eds.). Global Trends in Communication Education and Research. Cresskill, NJ: Hampton Press.

73. Wyss, V. (2002). Redaktionelles Qualitätsmanagement. Ziele, Normen, Ressourcen. Konstanz: uvk-Medien.

74. Zelizer, B. (2009). Why journalism's changing faces matter. En B. Zelizer (Ed.). The changing faces of journalism: Tabloidization, technology and truthiness. New York: Routlege. 